Instituto Internacional de Investigación y Desarrollo Tecnológico Educativo INDTEC, C.A.

DOI: https://doi.org/10.29394/scientific.issn.2542-2987.2017.2.3.18.325-342

OAI-PMH: http://www.indteca.com/ojs/index.php/Revista Scientific/oai

\title{
Estrategias Gerenciales de Calidad y Productividad: Dos Opciones para la Gerencia Educativa
}

\author{
Autora: Sonia María Aguilar Rondón \\ Universidad Nacional Experimental Rafael María Baralt, UNERMB \\ sonia aguilar1965@hotmail.com \\ Trujillo, Venezuela
}

\section{Resumen}

El Objetivo de este artículo es determinar las estrategias gerenciales de calidad y productividad para mejorar la gerencia educativa en la Educación Universitaria para ofrecer una educación de calidad y excelencia en la formación integral del individuo, considerando una serie de elementos, como: toma de decisiones, liderazgo, motivación, búsqueda de la excelencia. Este estudio se basó en la investigación documental y se llegó a la siguiente conclusión: es necesario una estructura de apoyo para implementar políticas, establecer normas, distribuir recursos y medir los resultados de aprendizaje de modo que se logre el mejor impacto posible sobre un aprendizaje productivo y de calidad para todos.

Palabras clave: gestión educativa; gerente educativo; liderazgo
cial. gerencial. 


\section{Management Strategies of Quality and Productivity: Two Options for Educational Management}

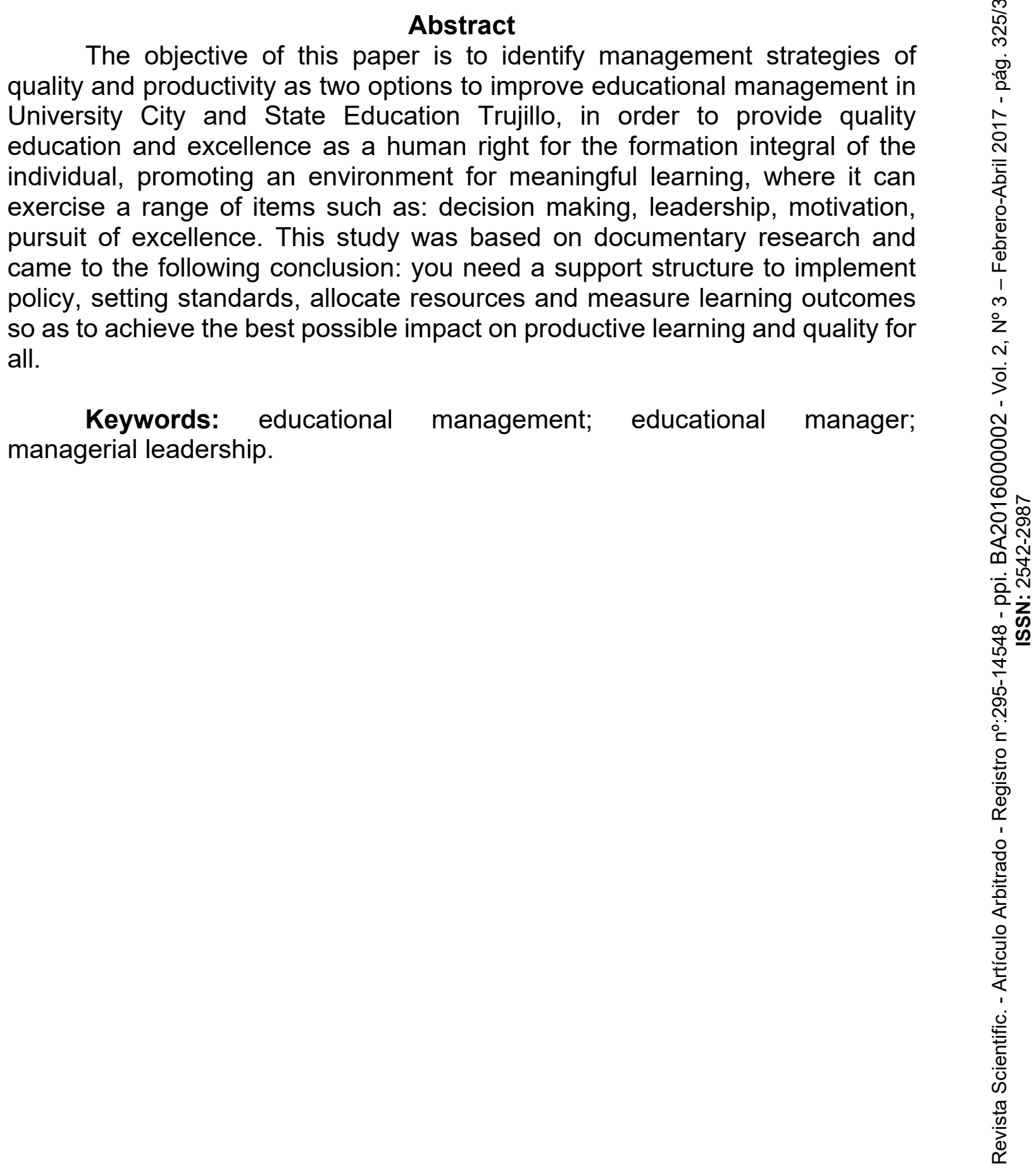

Date Received: 20-10-2016

Date Acceptance: 12-11-2016 


\section{Introducción}

Dada la importancia que reviste la educación universitaria para el proceso de formación del individuo y la capacitación de los recursos humanos requeridos para el desarrollo del país, la búsqueda de la excelencia y calidad en el producto, debe ser la premisa que oriente a relación su instrumentación e implementación para los diferentes niveles del sistema educativo.

Este ideal guarda relación, con los fines establecidos por la Constitución de la República Bolivariana de Venezuela (1999) y la Ley Orgánica de Educación (2009) donde establece que la educación venezolana es obligatoria y constituye una responsabilidad para el Estado, los padres y representantes y para cada docente. En los tiempos actuales, cuando la educación venezolana atraviesa una profunda situación de debilidad motivado a lo erróneo de las estrategias de gerencia aplicadas y a la falta de procesos administrativos sustentada en criterios científicos y ajena a posiciones subjetivas e interés sectoriales.

El presente estudio es innovador, pues relaciona el concepto de calidad total al ámbito educativo, busca constituirse en un aporte valido para motivar un discusión en el ámbito de docentes y personal directivo, la cual permita adquirir fortalezas suficientes tendientes a canalizar una revisión global de la forma como se ha venido aplicando el proceso de gerencia educativa y aprendizaje en las diferentes instituciones, y, posteriormente, aplicar estrategias que permitan lograr la calidad en el proceso. En tal sentido, la investigación se apoya en un tema innovador el concepto de la gerencia moderna, partiendo de la noción de calidad, busca aplicar tal criterio de gerencia y productividad al mejoramiento de la educación impartida en las instituciones de la entidad regional, específicamente, del Municipio Trujillo.

Así se tiene, que la investigación reviste importancia para el proceso educativo de los alumnos, considerando que estos representan el "producto" obtenido a partir de la operacionalización de los planes y programas 
desarrollados en cada institución y si se optimiza la calidad del proceso mediante el cual se cumple su proceso de aprendizaje más objetivo y adecuado a las necesidades que lo definen desde el punto de vista personal y social.

Dentro de este contexto, la figura del gerente adquiere un rol preponderante como capaz de motivar una matriz de transformación, la cual involucre a todos los elementos del ámbito educativo, fundamentalmente los referidos a la función administrativa, aplicando el nuevo precepto gerencial de "ganar- ganar", para adecuarla a los nuevos paradigmas de calidad, exigidos por el proceso de transformación socio-económica iniciados en el país, en el cual, por supuesto, involucra también la educación.

Sobre la base de la anterior premisa, el presente estudio tiene como objetivo esencial la revisión de los conceptos de calidad y productividad en el desempeño gerencial de la educación universitaria para determinar las estrategias gerenciales de calidad y productividad. La literatura consultada permitió fundamentar la investigación sobre bases teóricas objetivas, que luego a su vez, establecen las relaciones pertinentes.

\section{EI Problema}

La calidad y la productividad en las organizaciones dependen, en gran medida, del recurso humano que las mismas posean. La calidad es el máximo de eficiencia operativa tanto en el proceso de producción de los bienes, como en las relaciones interpersonales e inter-institucionales establecidas para alcanzar las metas y objetivos propuestos. La productividad, es la relación existente entre la cantidad de insumos invertidos en la elaboración de un producto y el resultado obtenido o el producto mismo. Según, Páez (1993): "cada día es más evidente que los recursos humanos, su inteligencia y conocimientos, así como el manejo de la información y disposición para el 
aprendizaje, constituyen elementos requeridos para la búsqueda de la calidad en cualquier organización" (págs. 92-93).

Más adelante, Páez (ob.cit): expone que la "calidad es sinónimo de competitividad eficiente, del logro de resultados óptimos" (pág. 103). En consecuencia, la competitividad del país depende de la calidad de los recursos humanos con que cuente, de su capacidad para generar ideas innovadoras y proponer, por supuesto, alternativas de solución creativas a los problemas confrontadas en cada uno de los ámbitos de la sociedad. No sólo para resolverlo momentáneamente, sino para erradicar definitivamente el foco perturbador.

Es por ello, que Deming (1986): afirma que países como Japón, Alemania, España, Francia, entre otros, presentan ventajas altamente competitivas, pues sustentan las mismas en la calidad de sus recursos humanos y su capacidad de gerencia, que es en realidad con lo que objetivamente cuentan, en comparación con otros países como Venezuela, los cuales poseen abundantes recursos materiales y minerales. Sin embargo, no logra un adecuado nivel competitivo, debido a que su recurso humano no responde a las exigencias, que tal proceso plantea y su capacidad técnica y expectativas están muy por debajo de las existentes en los citados países. En ese contexto, el aprendizaje y desarrollo de habilidades, igual que el conocimiento, constituye la mejor inversión para desarrollar las ventajas competitivas de cualquier organización.

Por esto, la búsqueda de la calidad requiere de una articulación armoniosa de los elementos de la organización, siendo ésta un sistema integrador de esfuerzos particulares y colectivos hacia la eficiencia del proceso productivo y la calidad del producto a un costo mínimo, no se logra sino es con la participación decidida de todos los miembros de la misma y la adopción de una nueva cultura gerencial y operacional. Por lo tanto, la educación debe estar presente, permanentemente, en el proceso de la búsqueda de la calidad. 
Cabe destacar, que la opinión de Páez (ob. cit), quien plantea: "la importancia estratégica de la educación, la formación y desarrollo del conocimiento y su impacto en la competitividad permite explicar la baja productividad y prosperidad experimentada por Venezuela en la última década".

Se infiere, a partir de la opinión de Páez, (ob.cit): que el sistema educativo venezolano no está identificado con la búsqueda de excelencia y la calidad total requerida en el producto, de igual modo, para gerenciar de manera eficiente los recursos que el país posee, se hace necesario, la formación de un hombre diferente, de un gerente potencial más que un administrador. Deming (ob.cit), establece que: "los sistemas y procesos tienen que cumplir lo indicado en los procedimientos y la norma, se pretende alcanzar unos objetivos de calidad. La calidad total es una filosofía, se busca la inexistencia de errores, los procesos se deben hacer bien a la primera" (pág. 98).

Así mismo, en las organizaciones universitarias, el personal como gerente le corresponde promover estrategias efectivas para la conducción de un personal al máximo en el logro de la productividad, adecuando sus estructuras gerenciales a la filosofía de calidad total, sobre la base de unos objetivos innovadores, vinculando al contexto socio-cultural-ambiental de la que circunscribe. De lo contrario, puede generar conflicto entre los participantes y la baja productividad. Es, por esto, que se hace necesario contar con un buen proceso de dirección institucional que dirija y asesore en sus aspectos administrativos y fundamentalmente lo impulse a la búsqueda de la excelencia.

Sin embargo, a lo expuesto anteriormente, es posible que el gerente carezca de factores elementales para optimizar al máximo los recursos disponibles y las potencialidades de cada uno de los integrantes de la organización. Entre estos factores se puede mencionar falta de liderazgo, toma de decisiones centralizadas, barreras de la comunicación, incorrecto manejo 
de problemas organizacionales y falta de motivación en el personal de la organización, que incide negativamente en la excelencia de los recursos humanos y en la calidad de los procesos administrativos puesto en práctica.

De esta manera, se torna interesante investigar lo que está ocurriendo en las instituciones universitarias del municipio y Estado Trujillo, sobre todo, en el desempeño de la función directiva. Sobre la base de estos elementos, el problema, en términos investigación, se formula a través de las interrogantes siguientes: ¿Cuáles son las estrategias gerenciales aplicadas por el gerente de las Instituciones Universitarias para impulsar la calidad y los factores de productividad?, ¿Cuál es la percepción que tienen los docentes de las estrategias gerenciales para impulsar la calidad y los factores de productividad? y ¿Cuál es la percepción que tienen los gerentes sobre su trabajo como gerencia?.

\subsection{Bases Teóricas}

\subsubsection{Calidad y Productividad}

A continuación, se presentan los conceptos y puntos de vista planeados por diversos autores acerca de la productividad y la calidad. Sin embargo, desde el punto de vista teórico, la investigación se centró en los planteamientos de Williams Edwards Deming, pionero y promotor de gran cantidad de elementos que aporta sobre la calidad total y la importancia de la gerencia en las organizaciones. En su obra Modelo Gerencial (1986) menciona catorce principios fundamentales para la calidad total:

(1) Creación y comunicación de la visión, el propósito y la misión de la organización para mejorar productos o servicios. Esto significa crear un plan para permanecer dentro del negocio ya sea a corto, mediano o largo plazo.

(2) Aprendizaje y adopción de la filosofía gerencial. Hace referencia poder entrar a la nueva era económica, a través de un liderazgo de cambio. El 
cambio de cultura no es fácil, y se lleva tiempo, pero sólo la alta gerencia puede lograrlo.

(3) Evitar el someter a los sujetos a la inspección masiva como medida de motivación para la productividad. La intervención siempre es tardía, ineficaz y costosa. El objetivo de este proceso es la auditoria para detectar cambios y comprobar medidas preventivas.

(4) El precio no será el único factor que se considere para realizar una compra. La competitividad de un producto está basada en las necesidades del cliente, se basa en la confiabilidad de los productos.

(5) Perfeccionar, mediante la mejora continua de la calidad los sistemas de producción y de servicio. Esto debe hacerse de una forma constante y permanente para poder mejorar la calidad y productividad para reducir los errores y desperdicios en los productos.

(6) Establecer la capacitación en el trabajo. Uno de los principales problemas en entrenamiento, y la supervisión es que no se han fijado estándares para poder medir cuál es un trabajo aceptable y cuál no lo es, de esta manera, el objetivo del supervisor es obtener determinada cantidad y calidad de producción.

(7) Promover, enseñar e instituir el liderazgo. La supervisión es responsabilidad de la administración y debe de eliminar las barreras que le impidan al trabajador desarrollar sus actividades.

(8) Combatir el temor propiciando un clima organizacional que favorezca la motivación. El miedo ira desapareciendo en la medida en que la administración, incluso los gerentes se vuelva un apoyo para sus trabajadores e inspiren confianza en ellos.

(9) Derribar las barreras que existen entre las diferentes áreas de la organización Este explica que entre departamentos deben de conocerse muy ampliamente y saber qué es lo que afecta a un departamento. 
(10) Eliminar los lemas, exhortaciones y metas meramente numéricas para los trabajadores Es muy importante eliminar todo tipo de slogan que impliquen la perfección, o un nuevo nivel de producción sin proponer como lograrlo, El proponer metas, sin un método para llegar a ellas, va a producir más efectos negativos que positivos.

(11) Eliminar los estándares de producción y las cuotas numéricas, sustituyéndolos por el mejoramiento continuo de la calidad. Normalmente estos estándares y metas numéricas vienen a sustituir al liderazgo.

(12) Eliminar los obstáculos que conspiren contra el orgullo de los trabajadores por haber realizado un buen trabajo. Un trabajador debe sentirse orgulloso de su trabajo, estar enterado cuando está bien o no.

(13) Instituir programas robustos de educación y reentrenamiento. Es necesario capacitar al personal en cuanto al uso de las estadísticas, para poder incorporar algunos sencillos métodos para que los empleados puedan llevar el control diario. El proceso de capacitación es sencillo, y puede hacerse en todos los niveles.

(14) Emprender acciones para alcanzar transformación organizacional. La administración necesitará la orientación de algún experto, pero este no asumirá la responsabilidad que le compete a la administración.

Analizando la postura de Deming, éste se refería a la calidad a todo aquello que le hiciera ahorrar a la empresa, y, a su vez, cumplir a tiempo con la entrega del producto al cliente, lo que ocasionó un revuelo en la manera de pensar de todos los gerentes al decirles que era más barato hacer un producto nuevo que corregirlo, ya que esto conlleva a la pérdida de tiempo y esfuerzo. Igualmente, recalcó que la producción falla porque se debe aplicar para mejorar de forma continua y contribuir a la satisfacción del cliente.

De esta forma, es importante en el campo educativo aplicar estrategias de calidad y productividad para un mejor desempeño de la gerencia del 
docente dentro del aula y una formación integral del individuo universitario que permita mayor competitividad y toma de decisiones adecuadas en su desarrollo como profesional, arrojando resultados favorables en su nivel de calidad y productividad, desde el punto de personal, pero, a su vez, logrando el éxito del organismo o empresa donde trabaje.

Según, Crosby (1990): a fines del siglo XIX, surge el sistema industrial, modelo en los Estados Unidos, "Frederick Taylor fue el pionero de la Administración Científica, puso en manos de los ingenieros industriales la planificación de trabajo, eximiendo de esta responsabilidad de los capataces" (pág. 87). En el siglo XX trajo consigo, de acuerdo con Rondón (1993): una era tecnológica, la cual permitió que las masas obtuvieran productos hasta entonces reservados sólo para los capitalistas.

Acerca de la capacitación para la búsqueda de la calidad y la productividad, han escrito varios autores. Según Gitlow y Gitlow (1998), plantea que: "la estructura organizacional interna da origen a barreras entre áreas del departamento" (pág. 61); la competencia, los rencores personales, las diferentes maneras de mirar un problema y las diferentes prioridades, constituyen barreras frecuentes, que obstaculizan la comunicación entre los departamentos y entre las áreas de estos (pág. 132).

Para Walton (1992): "la educación y el entrenamiento debe preparar a la gente para asumir nuevos cargos y responsabilidades" (pág. 73). Es importante tener presente la definición de calidad educativa propuesta por las Organizaciones de Naciones Unidas para la Educación, la Ciencia y la Cultura (OREAL- UNESCO SANTIAGO, 2007), dice "la educación de calidad, en tanto derecho fundamental de todas las personas, tienen como cualidades esenciales el respeto de los derechos, la equidad la relevancia y la pertinencia y dos elementos de carácter operativo: la eficacia y la eficiencia" (pág. 118). 


\subsubsection{Estrategias de Calidad y Productividad del Proceso Gerencial en}

las Instituciones Educativas.

Según, Deming (ob.cit): existen siete estrategias que contribuyen a la calidad y productividad del proceso gerencial en las instituciones educativas, a saber:

(1) Liderazgo: El gerente debe promover la horizontalidad en las comunicaciones, eliminando barreras jerárquicas y promover la integración que conlleva a una interacción cara a cara, personalizada, a fin de unificar esfuerzos hacia la solución de los problemas. Por supuesto, debe ser un excelente planificador y previsor de situaciones futuras. Hacer énfasis en el propósito de mejorar los productos y el servicio con la declaración de la visión y misión organizacional.

(2) Comunicación: El gerente debe utilizar la interacción comunicativa con los demás integrantes de la organización con el propósito de facilitar la consolidación de una visión compartida y capacitación constante de los recursos humanos de acuerdo con las responsabilidades que les corresponde asumir durante su desempeño en función del objetivo deseado. Comunicar permanentemente que la calidad no se logra mediante inspección sino mediante el mejoramiento del proceso. Eliminación de barreras comunicacionales e integrar acciones para el logro de la productividad.

(3) Solucionador de Conflictos: El gerente de calidad, debe manejar el conjunto de intereses particulares que interactúan en la organización y que momentos determinados, sobre el interés colectivo, ocasionando carga de desperdicio al proceso productivo. Debe promover la sinergia entre las fuerzas que coexisten en la institución facilitando su interacción armónica y efectiva. Eliminando las barreras entre los departamentos promoviendo trabajo en equipo, satisfacción de intereses personales y colectivos. El gerente debe seleccionar las estrategias más idóneas para facilitar la solución a la 
problemática planteada en un clima de armonía y solidaridad; de lo contrario, puede generar conflictos entre los participantes y baja productividad.

(4) Motivación: El gerente en la calidad de la Institución debe aprovechar cualquier oportunidad en la organización para incentivar la participación de los miembros de la comunidad hacia el trabajo productivo y hacia el crecimiento personal. el cumplimiento de la visión y misión, el mejoramiento de la calidad del producto y servicio, la integración en equipo y eliminar el desperdicio.

(5) Organización: implica el desarrollo de la gestión en las diferentes instituciones, facilitando la integración y coordinación de las actividades de los docentes, alumnos, y otros agentes; como el empleo de los recursos para desarrollar los procesos programas y proyectos, que involucran la división del trabajo y de funciones, a través de un a jerarquía de autoridad y responsabilidad.

(6) La dirección: Relacionada con el liderazgo, la motivación y la creación de un clima organizacional por parte del gestor. Que integra las potencialidades de los diferentes sujetos, a partir del compromiso de todos con el proyecto formativo para mejorar la docencia y la administración de los recursos de las instituciones.

(7) Toma de Decisiones: Constituye lo cotidiano en el desempeño del gerente de la calidad puesto que la dinámica de la institución, requiere del mismo un constante manejo de conflictos, situaciones, problemas, para satisfacer las expectativas y necesidades planteadas en cada uno de los miembros de la organización.

\subsubsection{La Productividad como Fórmula para el Éxito}

La actitud de superación constante, debe existir en todo y cada uno de los hombres que integran una organización. Si este se estanca y se encierra en su propio mundo sin querer asomarse a la realidad de un mundo cambiante, 
se privará de la posibilidad de transformar su propia manera de vivir y por supuesto, la del entorno que lo determina. En tal sentido, la educación juega un papel fundamental para la superación del individuo. Superación, que por supuesto, lo llevara alcanzar niveles más elevados en el proceso productivo donde interactúa. De este modo, la productividad genera satisfacción en el sentido de que el individuo está consciente de que contribuye a una función realmente efectiva, productiva, llevándolo esto a niveles cada vez más elevados de preparación, base esencial para obtener el éxito desde el punto de vista personal y social.

\subsubsection{Factores para Lograr la Productividad}

Según Hiba (2001), explica que existen tres factores que inciden en el logro de la productividad gerencial:

(1) Compartir los Beneficios: Que el éxito en la productividad no se quede represado en su persona, el mismo debe hacerse extensivo toda la organización de la cual forma parte, pues el resultado de la eficiencia productiva poco a poco va desfragmentando cada una de las dependencias de la misma, abriendo espacios cada vez más amplio para el logro de la calidad total en el producto generado. Así, la productividad no es un hecho aislado, es parte de un proceso integrado, con aristas bien definidas donde se requiera el aporte de cada uno de los miembros de la organización, la unión de los esfuerzos aislados en una función de un resultado global.

(2) Adoptar una filosofía de calidad en la productividad: Implica que el gerente, por lo menos, debe ganarse un proceso de apertura, de entender que la organización por sí misma, en forma aislada no puede marchar, sino que necesita integrarse a un sistema más complejo que es definido por el entorno sociocultural, ambiental y económico que la determina.

(3) Satisfacer necesidades de su personal: Es decir comprender que los trabajadores requieren actualización para alcanzar mejores niveles de 
producción y calidad en el proceso, satisfacer sus necesidades desde el punto de vista personal y social (como integrantes de la organización).

\subsubsection{La Calidad Total y la Productividad en la Educación Universitaria}

La búsqueda de la calidad total en educación es un proceso que requiera una total disposición a la apertura y al cambio de parte de cada uno de los miembros integrantes de las instituciones educativas. Productividad es educación, no es solamente rendimiento académico. No se puede pensar según, Martínez (2004): "en la posibilidad de alcanzar la calidad total si uno de los componentes del sistema funcional de la institución no responde a las expectativas del resto de componentes". Es decir, la calidad total no es, un proceso aislado, al contrario, la productividad optimizada requiere del concurso de todas las personas que definen la institución, desde los estudiantes, docentes, y demás personas vinculada otro modo a la misma.

El paradigma de la productividad y calidad en educación, no se basa sólo en la estructura organizacional, ni en el estilo y los sistemas gerenciales, sino en la eficacia de los docentes y otros miembros de la institución. Se reconoce que las personas constituyen el valor supremo de la organización, porque son los programadores, los que producen todo lo demás en los niveles: personal, interpersonal, gerencial y organizacional, de los docentes y demás miembros, que interactúan en la institución.

Por otra parte, para alcanzar dicha productividad, es necesaria la adecuada formación y entrenamiento de los recursos humanos. La productividad amerita el reclutamiento de personal cuidadosamente seleccionado, pues es conciliando su potencial creativo, laboral y aptitudinal como se logra la optimización del proceso desarrollado hacia la búsqueda de la excelencia y calidad deseada. 


\section{Metodología}

En toda indagación, a juicio de Malavé (2003): se describe que la metodología "es el fundamento para el desarrollo del trabajo de exploración propiamente dicho" (pág. 69). De lo señalado, es importante destacar que la metodología, de acuerdo a Palella y Martins (2004): "es el estudio crítico del método" (pág. 73).

Según, la fuente de información o la estrategia utilizada, puede decirse que el artículo presentado reviste características de investigación documental y de investigación descriptiva, simultáneamente. Al respecto, Tamayo y Tamayo (2007), señala que: "los tipos de investigación difícilmente se presentan puros generalmente se combinan entre sí y obedecen sistemáticamente a la aplicación de la investigación" (pág. 32).

Por otra parte, Sierra (2003): define la investigación documental como aquella que "tiene por objeto directo la observación de fuentes documentales" (pág. 35). En otras palabras, se ha demostrado el proceso de búsqueda y proceso de información formado a partir de estudios creados sobre este particular, acumulados en el transcurso de la historia de la humanidad. Esta investigación por tanto es descriptiva, en lo que respecta al objetivo de analizar los factores y estrategias aplicadas por el gerente para mejorar la calidad y productividad en la Educación Universitaria.

\section{Conclusiones}

El estudio realizado fue con el propósito de analizar los factores y estrategias aplicadas por el gerente para mejorar la calidad y productividad en la Educación Universitaria, arrojando como resultado las siguientes conclusiones:

- La calidad y la productividad son elementos esenciales de la educación universitaria, que no sólo tiene repercusiones en lo que aprenden 
los estudiantes, sino también en su manera de formarse y en los beneficios que obtienen de la instrucción que reciben.

- Es fundamental la búsqueda de medios, para lograr que los alumnos obtengan resultados académicos dignos y adquieran valores y competencias que le permitan desempeñar un papel positivo en la sociedad.

- En la gerencia es sumamente importante la búsqueda de la calidad, al indicar que la excelencia requiere de una articulación armoniosa, de los elementos de la organización, integrando esfuerzos particulares y colectivos hacia la productividad.

- El gerente educativo como el gestor de la calidad debe manejar diversas estrategias gerenciales para optimizar los procesos de calidad y productividad, por ejemplo, debe ser líder, tomar decisiones acertadamente, poseer buena comunicación con los subordinados, entre otras.

- Todo aprendizaje debe darse desde la aprehensión de la realidad, es decir, apropiarse del objeto del conocimiento y ponerlo en práctica de forma creativa. Debido a esto, los aprendizajes se pueden obtener de manera creativa, participativa, interactiva, recreativa, trabajados en equipo, reflexionando que los mismos forman parte del acontecimiento para lograr una alineación y un progreso humano integral.

Con esto, es posible registrar algunos símbolos que marcan la aparición de una gerencia para nuevos tiempos, es decir la que coloca al Ser Humano como un servidor en los espacios actuales llamados postmodernos, que conceptúa al Ser Humano, como pensante, sintiente, todo enfocado hacia una nueva intencionalidad.

\section{Referencias}

Constitución de la República Bolivariana de Venezuela (1999). Gaceta Oficial Extraordinaria $\mathrm{N}^{\circ} \mathbf{3 6 . 8 6 0}$ de fecha 30 de diciembre. Venezuela: Asamblea Nacional Constituyente. 
Crosby, P. (1990). Hablemos de Calidad. México: McGraw-Hill.

Deming, E. (1986). Propuesta de un Plan de Mejora Continua para una Empresa de Jardinería. Capítulo II: Marco Teórico. Universitas Americarum.

Gitlow, H. y Gitlow, S. (1998). Como Mejorar la Calidad y la Productividad con el Método Deming. Bogotá: Editorial Norma.

Hiba, J. (2001). Mayor productividad y un mejor lugar de trabajo: estrategias y resultados en América Latina de un método activo de capacitación empresarial de pequeñas y medianas empresas. OIT, Lima.

Ley Orgánica de Educación (2009). Gaceta Oficial No 5.929 Extraordinario del 15 de agosto. República Bolivariana de Venezuela: La Asamblea Nacional.

Malavé, $\mathrm{P}$ (2003). El régimen tributario constitucional en comentarios a la CRBV. Caracas, Venezuela: VADEL Hermanos Editores.

Martínez, A. (2004). De la Escuela Expansiva a la Escuela Competitiva. Dos modos de modernización educativa en América Latina. España: Anthropos Editorial.

Páez, T. (1993). Calidad y Productividad en el Sistema Educativo. Revista Calidad y Productividad. Caracas.

Palella, S. y Martins, F. (2004). Metodología de la investigación cuantitativa. Caracas: Fondo Editorial FEDUPEL.

Rondón, G. (1993). Calidad Total. Caracas, Venezuela.

Sierra, R. (2003). Técnicas de investigación social. España: Editorial Siglo $\mathrm{XXI}$.

Tamayo, y Tamayo, M. (2007). El proceso de la investigación científica. Cuarta edición. Limusa. México.

UNESCO (2007). La conceptualización de la UNESCO sobre calidad: un marco para el entendimiento, el monitoreo y la mejora de la calidad educativa. Herramientas de Formación para el Desarrollo Curricular. UNESCO-OIE.

Walton, M. (1992). ¿Cómo Administrar con el Método Deming? Bogotá, Colombia. 


\section{Sonia María Aguilar Rondón}

e-mail: sonia aguilar1965@hotmail.com

Nacida en Valera, estado Trujillo, Venezuela. Actualmente cursa estudios de Doctorado en Ciencias de la Educación en la Universidad Nacional Experimental "Rafael María Baralt". Maestría en Administración de la Educación Básica Magíster Scientiarum 1994-1997; Diplomado en Cooperativismo y Gestión Comunitaria 2007 en el Instituto Universitario de Tecnología del Estado Trujillo; Curso de: Didáctica Especial en el Área de Castellano y Literatura, Matemática y Física y en el Área de Biología y Química 160 Horas 2010 (Diplomado) en el Liceo Bolivariano Antonio José Pacheco Estado Trujillo, convenio Cuba Venezuela. Experiencia Laboral actualmente Prof. Agregado de la Universidad Politécnica Territorial "Mario Briceño Iragorry".

El contenido de este manuscrito se difunde bajo una Licencia de Creative Commons ReconocimientoNoComercial-Compartirlgual 4.0 Internacional 